Article

\title{
Analytical Study for the Charge-Transfer Complexes of Rosuvastatin Calcium with $\pi$-Acceptors
}

\author{
Nourah Z. Alzoman ${ }^{1}$, Maha A. Sultan ${ }^{1}$, Hadir M. Maher ${ }^{1,2}$, Mona M. Alshehri ${ }^{1}$, \\ Tanveer A. Wani ${ }^{1}$ and Ibrahim A. Darwish ${ }^{1, *}$
}

1 Department of Pharmaceutical Chemistry, College of Pharmacy, King Saud University, P.O. Box 2457, Riyadh 11451, Saudi Arabia; E-Mails: nalzoman@ksu.edu.sa (N.Z.A.); mahasultan59@gmail.com (M.A.S.); hadirrona@yahoo.com (H.M.M.); abs.office1@yahoo.com (M.M.A.); tanykash@yahoo.co.in (T.A.W.)

2 Department of Pharmaceutical Analytical Chemistry, Faculty of Pharmacy, University of Alexandria, El-Messalah, Alexandria 21521, Egypt

* Author to whom correspondence should be addressed; E-Mail: idarwish@ksu.edu.sa; Tel.: +966-11-467-7348; Fax: +966-11-467-6220.

Received: 31 May 2013; in revised form: 18 June 2013 / Accepted: 21 June 2013 /

Published: 3 July 2013

\begin{abstract}
Studies were carried out to investigate the charge-transfer (CT) reaction of ROS-Ca, as a n-electron donor with various $\pi$-acceptors: tetracyanoethylene, $p$-chloranilic acid, 2,3-dichloro-5,6-dicyano-1,4-benzoquinone, 2,3,5,6-tetrabromo-1,4-benzoquinone, 1,3,5trinitrobenzene, 2,3,5,6-tetrachloro-1,4-benzoquinone, 7,7,8,8-tetracyano-quinodimethane, and 2,4,7-trinitro-9-fluorenone. Different colored CT complexes were obtained. The reaction mechanism and site of interaction were determined by ultraviolet-visible spectrophotometric techniques and computational molecular modeling. The formation of the colored complexes was utilized in the development of simple, rapid and accurate spectrophotometric methods for the determination of ROS-Ca. Under the optimum reaction conditions, linear relationships with good correlation coefficients (0.9984-0.9995) were found between the absorbances and the concentrations of ROS-Ca in the range of $2-200 \mu \mathrm{g} \mathrm{mL}{ }^{-1}$. The limits of detection ranged from 0.41 to $12.24 \mu \mathrm{g} \mathrm{mL}^{-1}$. No interference could be observed from the additives commonly present in the tablets or from the drugs that are co-formulated with ROS-Ca in its combined formulations. The methods were successfully applied to the analysis of tablets with good accuracy and precision; the recovery percentages ranged from $99.54-100.46 \pm 1.58-1.82 \%$. The results were compared favorably with the reported method. The proposed methods are practical and valuable for
\end{abstract}


routine application in quality control laboratories for determination of ROS-Ca in its bulk form and tablets.

Keywords: atherosclerosis; cholesterol; rosuvastatin calcium; charge-transfer complexes; spectrophotometry; pharmaceutical analysis

\section{Introduction}

Rosuvastatin calcium (ROS-Ca, bis[(E)-7-[4-(4-fluorophenyl)-6-isopropyl-2-[methyl(methylsulfonyl)-amino] pyrimidin-5-yl](3R,5S)-3,5-dihydroxyhept-6-enoic acid calcium salt, Figure 1) is a synthetic 3-hydroxy-3-methylglutaryl-coenzyme A (HMG-CoA) reductase inhibitor. It exerts its action by specifically inhibiting the HMG-CoA reductase, the enzyme that catalyzes the conversion of HMG-CoA to mevolanate, which is the early rate-limiting step in the biosynthesis of cholesterol in the body. Inhibition of the enzyme decreases de novo cholesterol synthesis, increasing expression of low-density lipoprotein (LDL) receptors on hepatocytes. This increases the uptake of LDL by the hepatocytes, decreasing the amount of LDL-cholesterol in the blood. ROS-Ca also reduces blood levels of triglycerides and slightly increases levels of HDL-cholesterol [1-3].

The therapeutic importance of ROS-Ca was behind the growing interest in the development of analytical methods for its determination in its bulk and pharmaceutical dosage forms. A literature survey revealed that several analytical methods were reported for ROS-Ca determination. These methods include high-performance thin-layer chromatography [4], High performance liquid chromatography (HPLC) [5,6], and capillary zone electrophoresis [7].

HPLC is an efficient analytical technique and it is widely applied in pharmaceutical analysis; however it relies on expensive instrumentation that is not available in most quality control laboratories and time-consuming procedures in establishing the most appropriate chromatographic conditions. In general, spectrophotometry is the most widely used technique in pharmaceutical analysis because of its inherent simplicity and wide availability in most quality control laboratories [8-13]. For these reasons, spectrophotometric method for determination of ROS-Ca is required as an alternative for HPLC. The spectrophotometric methods that have been reported for determination of ROS-Ca suffer from major drawbacks [14-17]. These drawbacks include decreased selectivity due to measuring the native light absorption of ROS-Ca in the blue-shifted ultraviolet region, which might be subjected to interferences, employment of multiple-steps of non-selective oxidation reactions and tedious liquid-liquid extraction procedures using large volumes of organic solvents in the methods based on formation of ion-pair associates. Therefore, the development of new alternative spectrophotometric methods for determination of ROS-Ca in its bulk form and pharmaceutical dosage forms (tablets) is very essential.

The molecular interactions between the electron-donating pharmaceutical compounds and electron-accepting reagents are generally associated with the formation of intensely colored CT complexes, which usually absorb radiations in the visible region. The rapid formation of these complexes leads to their widespread utility in the development of visible spectrophotometric methods for analysis of many pharmaceutical compounds [18-25]. Literature survey revealed that the CT reaction of ROS-Ca has not been investigated yet. This fact promoted our interest in employment of 
the CT-reaction as a basis for the development of new spectrophotometric methods for determination of ROS-Ca.

\section{Results and Discussion}

\subsection{Spectral Characteristics of the Reaction}

The interaction of ROS-Ca (Figure 1) with polyhaloquinone and polycyanoquinone $\pi$-acceptors (Figure 2) in non-polar solvents such as dichloroethane was found to produce colored CT complexes with low molar absorptivity values.

Figure 1. Chemical structures of rosuvastatin calcium (ROS-Ca) and the co-formulated drugs.
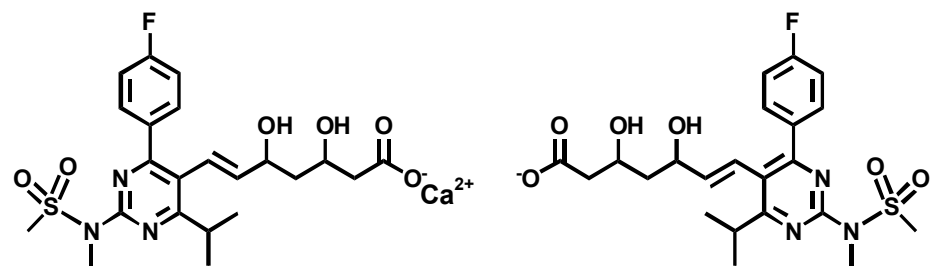

Rosuvastatin calcium (ROS-Ca)<smiles>CC(C)CC(=O)C(C)(C)Oc1ccc(C(=O)c2ccc(Cl)cc2)cc1</smiles>

Fenofibrate<smiles>O=C(CCC(O)c1ccc(F)cc1)N(c1ccc(O)cc1)c1ccc(F)cc1</smiles>

Ezetimibe

Figure 2. Chemical structures of the polyhaloquinone and polycyanoquinone $\pi$-acceptors used in the present study.<smiles>O=C1C(=O)C(Cl)C(=O)C(Cl)=C1O</smiles>

p-Chloranilic acid (pCA)<smiles>O=[N+]([O-])c1cc([N+](=O)[O-])cc([N+](=O)[O-])c1</smiles>

1,3,5-Trinitrobenzene (TNR)<smiles>N#Cc1ccc(C(C#N)C#N)cc1</smiles>

7,7,8,8-Tetracyanoquinodimethane (TCNQ)<smiles>O=C1C(Br)=C(Br)C(=O)C(Br)=C1Br</smiles>

Bromanil<smiles>N#CC1=C(C#N)C(=O)C(Cl)=C(Cl)C1=O</smiles>

2,3-Dichloro-5,6-dicyano-1,4-be

nzoquinone (DDQ)

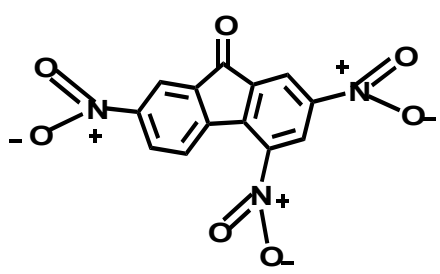

2,4,7-Trinitro-9-fluorenone (TNF) 
In polar solvents such as methanol or acetonitrile, complete electron transfer from the ROS-Ca (D), as an electron donor, to the acceptor moiety (A) takes place with the formation of intensely colored radical ions with high molar absorptivity values, according to the following scheme:

$$
\begin{aligned}
& \text { polar } \\
& \mathrm{D}+\mathrm{A} \rightleftharpoons \begin{array}{c}
(\mathrm{D}-\mathrm{A}) \stackrel{\text { solvent }}{\text { complex }} \mathrm{D}^{++}+\mathrm{A}^{--} \\
\text {radical ions }
\end{array}
\end{aligned}
$$

The dissociation of the (D-A) complex was promoted by the high ionizing power of the polar solvent and the resulting peaks in the absorption spectra of ROS-Ca-acceptor reaction mixtures were similar to the maxima of the radical anions of the acceptors obtained by the iodide reduction method [26].

The interaction of ROS-Ca with $\pi$-acceptors at room temperature gave colored chromogens showing different absorption maxima at 840, 435, 460, 518, 412, 498, 460, and $412 \mathrm{~nm}$ for TCNQ, TNB, DDQ, pCA, TCNE, bromanil, chloranil, and TNF, respectively (Figures 3-5).

The predominant chromogen with TCNQ in acetonitrile is the bluish-green colored radical anion, which exhibits strong absorption maxima at 840, 823, 760, and $740 \mathrm{~nm}$ (Figure 4). These bands may be attributed to the formation of the radical anion TCNQ, which was probably formed by the dissociation of an original donor-acceptor (D-A) complex with ROS-Ca. The dissociation of the complex was promoted by the high ionizing power of acetonitrile. Further support of this assignment was provided by the absorption maxima with those of TCNQ radical anion produced by the iodide reduction method [26].

The complex of ROS-Ca with TNB showed two absorption maxima at 435 and $550 \mathrm{~nm}$ (Figure 3). The intensity of the first maximum is about 1.5 fold the second one. Therefore, measurements were carried out at $435 \mathrm{~nm}$, at which higher sensitivity was achieved.

Figure 3. Absorption spectra of the CT complexes of ROS-Ca with TNB (1) and DDQ (2) Concentrations of ROS-Ca were 50 and $15 \mu \mathrm{g} \mathrm{mL}{ }^{-1}$ in case of TNB and DDQ, respectively. Solutions were prepared in acetonitrile for reaction with TNB and in methanol for reaction with DDQ.

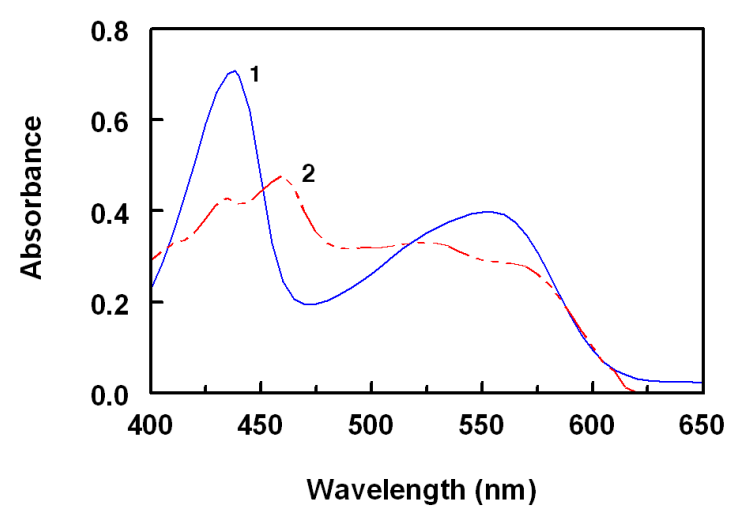

Chloranilic acid (pCA) exists in three ionic forms, the neutral yellow-orange $\mathrm{H}_{2} \mathrm{~A}$ at very low $\mathrm{pH}$, the dark purple $\mathrm{HA}^{-}$which is stable at $\mathrm{pH}=3$ and a pale violet $\mathrm{A}^{2-}$, which is stable at high $\mathrm{pH}$; these transformations are illustrated in the following scheme: 


$$
\begin{array}{ccc}
\mathrm{H}_{2} \mathrm{~A} & \rightleftharpoons \mathrm{H}^{+}+\mathrm{HA}^{-} \text {(violet) } \\
\mathrm{HA}^{-} & \rightleftharpoons \mathrm{H}^{+}+\mathrm{A}^{2-} \text { (colorless) }
\end{array}
$$

Since the interaction of ROS-Ca with pCA in acetonitrile gave a violet product (Figure 4), it might be concluded that HA was the form of pCA involved in the reaction described herein.

Figure 4. Absorption spectra of the CT complexes of ROS-Ca with TCNE (1), pCA (2), and TCNQ (3). Concentrations of ROS-Ca were 50, 70, and $20 \mu \mathrm{g} \mathrm{mL} \mathrm{m}^{-1}$ in case of TCNE, $\mathrm{pCA}$, and TCNQ, respectively. Solutions were prepared in acetonitrile for reaction with all acceptors.

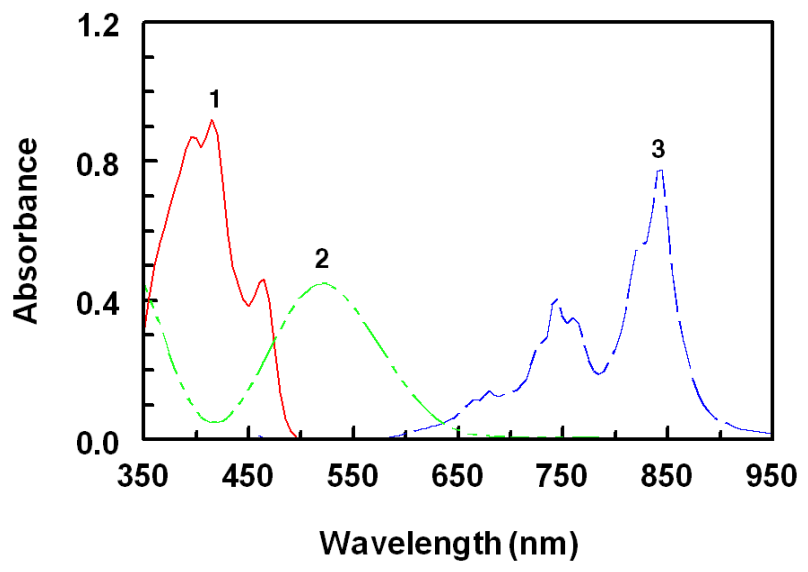

With TCNE, the characteristic shaped absorption band of TCNE radical anion with reported maximum in acetonitrile at $430 \mathrm{~nm}$ was not found (Figure 4). Instead, a duplet at 392 and $412 \mathrm{~nm}$ was formed which corresponds to the 1,2,3,3-pentacyanopropeneide (PCNP) anion, which is more preferable than TCNE anion, in quantitative analysis, in having higher molar absorptivity [26]. The resulting maxima of ROS-Ca with DDQ (Figure 3), bromanil, chloranil (Figure 5), and TNF are similar to that of radical anions of these acceptors obtained by the reduction method and coincide with the values reported in the literature $[27,28]$.

Figure 5. Absorption spectra of the CT complexes of ROS-Ca with bromanil (1) and chloranil (2). Concentrations of ROS-Ca were 80 , and $150 \mu \mathrm{g} \mathrm{mL}^{-1}$ in case of bromanil and chloranil, respectively. Solutions were prepared in acetonitrile for reaction with both acceptors.

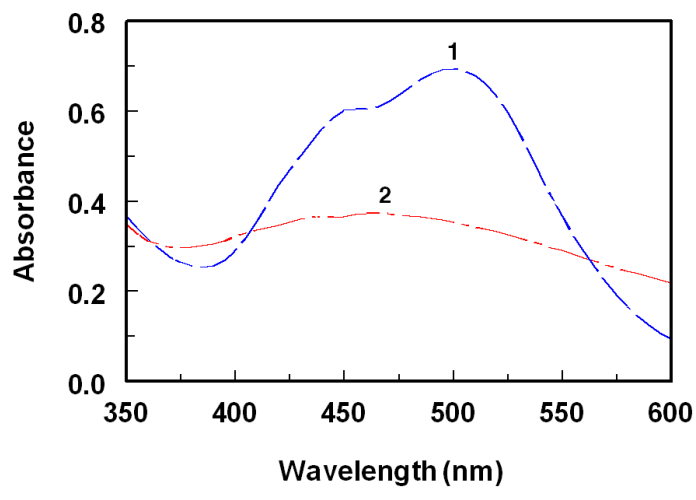


The relative sensitivity of the eight acceptors employed in the present analytical work may be attributed to their difference in electron affinities, as well as the conditions employed in the reaction (reagent concentration, reaction time, and solvent). Bromanil, chloranil, and TNF gave relatively weak molar absorptivity values (Table 1). This may be explained on the basis of insufficient ionization of these relatively weak $\pi$-acceptors that possess lower electron affinities than TCNQ and DDQ [29]. Because of the poor sensitivity of TNF and its very slow reaction, it has been excluded from further investigations.

\subsection{Optimization of Reaction Conditions}

The results of variations in the reagents concentrations indicated that $1 \mathrm{~mL}$ of the concentrations indicated in Table 1 were the optimum concentrations. The higher concentrations of the reagents may be useful for rapidly reaching equilibrium, thus minimizing the time required to attain maximum absorbance at the corresponding wavelengths of maximum absorbance.

In order to select the most appropriate solvent, the reactions were carried out in different solvents. Small shifts in the position of the maximum absorption peak were observed, and the absorption intensities were also influenced. Methanol gave maximum sensitivity in the case of DDQ and acetonitrile was considered as an ideal solvent for the other acceptors. This because it offered maximum sensitivity, which was attributed to the high dielectric constant of acetonitrile that promotes maximum yield of radical anions, in addition to its high solvating power for the acceptors [30].

The optimum reaction time was determined by monitoring the color development at room temperature $\left(25 \pm 2{ }^{\circ} \mathrm{C}\right)$. Complete color development was attained instantaneously with DDQ, and PCA, or after 5-60 min with other acceptors (Table 1). The developed colors remained stable at room temperature for at least a further $30 \mathrm{~min}$.

\subsection{Molar Ratio of the Reaction, Molecular Modeling, and Proposing the Site of Interaction}

Job's method of continuous variation was used for determining the molar ratio of ROS-Ca to DDQ. From the obtained Job's plot (data not shown), it was concluded that the ROS-Ca:DDQ ratio is 1:2. This indicated that two moles of DDQ interacted with one mole of ROS-Ca. Considering the divalent calcium ion, the reaction was postulated to proceed as 1:1 ratio for DDQ with rosuvastatin anion via only one site of interaction in spite of the presence of more than one possible electron-donating site. For investigating the site of interaction and postulate the reaction mechanism, modeling for the CT complex was performed. It was found that the highest electron densities in the rosuvastatin molecule are located on the two oxygen atoms of the sulfonamide group. The total charges on each of the two oxygen atoms of the sulfonamide anion were found to be -0.96543 and -0.97147 . As well, it was found that DDQ moves toward the sulfonamide group of ROS to form the CT complex (Figure 6). In the sulfonamide group, sulfur can donate a major share of the lone pairs of electrons to the two oxygen atoms. This leads to the development of partial negative charges on the oxygen atoms, and this makes them capable of forming the charge transfer complex. Other centers did not contribute in the CT reaction based on the fact that certain electron density was required for achievement of a successful electron transfer [29]. 
Table 1. Optimum conditions for the CT reaction of ROS-Ca with different $\pi$-acceptors and the achieved molar absorptivities.

\begin{tabular}{cccccc}
\hline \multirow{2}{*}{ Acceptor $^{\text {a }}$} & \multicolumn{3}{c}{ Condition } & \multicolumn{2}{c}{$\begin{array}{c}\text { Molar absorptivity } \\
\left(\boldsymbol{\varepsilon} \times \mathbf{1 0}^{-4}\right)\end{array}$} \\
\cline { 2 - 5 } pCA & Reagent conc. $\left(\mathbf{m g ~ m L}^{-\mathbf{1}}\right)$ & Solvent & Time (min) & $\boldsymbol{\lambda}_{\max }(\mathbf{n m})$ & 1.4 \\
DDQ (1.9) & 4 & Acetonitrile & At once ${ }^{\mathrm{b}}$ & 518 & 3.0 \\
TCNE (2.2) & 2 & Methanol & At once ${ }^{\mathrm{b}}$ & 460 & 1.8 \\
TNB (0.7) & 2 & Acetonitrile & 15 & 412 & 0.64 \\
TCNQ (1.7) & 4 & Acetonitrile & 30 & 435 & 4.0 \\
Bromanil (1.37) & 1 & Acetonitrile & 15 & 840 & 0.88 \\
Chloranil (1.37) & 5 & Acetonitrile & 5 & 498 & 2.2 \\
TNF (1.1) & 5 & Acetonitrile & 5 & 460 & 0.25 \\
\hline
\end{tabular}

${ }^{a}$ Figures in parenthesis are the electron affinities of the acceptors [29]. Electron affinity of pCA could not be found in the available literatures. ${ }^{\mathrm{b}}$ For getting readings with high precision, the reactions were allowed to proceed for $5 \mathrm{~min}$.

Figure 6. Energy-minimized CT complex of ROS-Ca with DDQ.

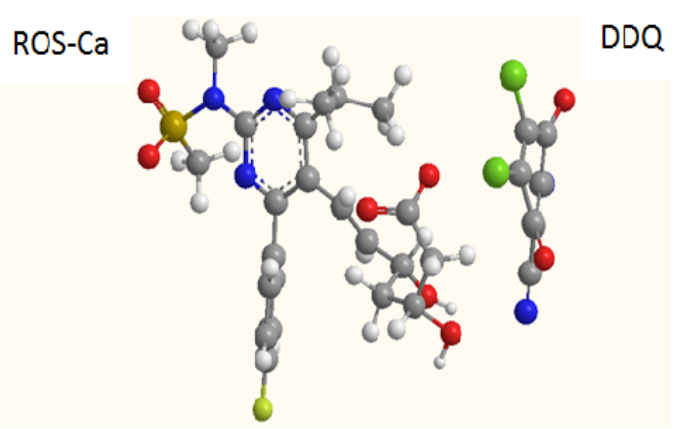




\subsection{Development and Validation of the Analytical Methods}

\subsubsection{Calibration Curves, Linearity and Sensitivity}

Under the specified optimum reaction conditions, the calibration curves for ROS-Ca with the different analytical reagents employed in the present work were constructed. The regression equations for the results were derived using the least-squares method. In all cases, Beer's law plots $(n=5)$ were linear with very small intercepts and good correlation coefficients in the general concentration range of 2-200 $\mu \mathrm{g} \mathrm{mL}^{-1}$ (Table 2). The limits of detection (LOD) and limits of quantitation (LOQ) were determined [31] using the formula: LOD or LOQ $=\kappa S D a / b$, where $\kappa=3$ for LOD and 10 for LOQ, $\mathrm{SDa}$ is the standard deviation of the intercept, and $\mathrm{b}$ is the slope. Based on the basis of 3 replicate measurements, the limits of detection were $0.41-12.24 \mu \mathrm{g} \mathrm{mL}^{-1}$.

\subsubsection{Precision}

The precisions of the assays (within-assay and between-assays) were determined at the ROS-Ca concentrations cited in Table 3. The within-assay precision was assessed by analyzing six replicates of each sample as a batch in a single assay run, and the between-assays precision was assessed by analyzing the same sample, as triplicate, in two separate assay runs. The assays gave satisfactory results; the relative standard deviations (RSD) were less than $2 \%$ (Table 3). This level of precision of the proposed methods was adequate for the quality control analysis of ROS-Ca.

\subsubsection{Specificity and Interference}

The proposed assay has the advantages that the measurements are performed in the visible region, away from the UV-absorbing interfering substances that might be co-extracted from dosage forms that contain ROS-Ca. The interference from the congenital drugs that is co-formulated with ROS-Ca in some of its dosage forms was studied. These drugs were fenofibrate [32] and ezetimibe [33]. The chemical structures of these drugs are given in Figure 1. Potential interferences of these drugs were studied in a ratio which is normally present in their combined dosage forms. No interferences from these drugs were found with ROS-Ca in the proposed assay. This selectivity of the CT reaction for ROS-Ca was attributed to its adequate basic character (electron-donating), which allowed the CT, rather than the other drug, that does not have the adequate basic property that is required to achieve CT reaction. As well, no interference was observed from the excipients with the proposed assay as indicated from the obtained good recovery (mentioned above). The absence of interference from the excipients, even though they contain basic component(s) was attributed to the extraction of the ROS-Ca tablets prior to the analysis with methanol in which the excipients do not dissolve. 
Table 2. Quantitative parameters for the determination of ROS-Ca based on its CT reaction with various $\pi$-acceptors.

\begin{tabular}{ccccccc}
\hline Acceptor & $\begin{array}{c}\text { Range } \\
\left(\boldsymbol{\mu g} \mathbf{~ m L}^{-\mathbf{1}}\right)\end{array}$ & Intercept & Slope & $\begin{array}{c}\text { Correlation } \\
\text { coefficient }\end{array}$ & $\begin{array}{c}\text { LOD } \\
\left(\mu \mathbf{g ~ m L}^{-\mathbf{1}}\right)\end{array}$ & $\begin{array}{c}\text { LOQ } \\
\left(\boldsymbol{\mu g} \mathbf{~ m L}^{-\mathbf{1}}\right)\end{array}$ \\
\hline TCNQ & $5-50$ & 0.0032 & 0.0388 & 0.9995 & 0.41 & 1.37 \\
TNB & $4-30$ & 0.0171 & 0.0137 & 0.9992 & 1.52 & 5.07 \\
DDQ & $2-40$ & 0.0146 & 0.0304 & 0.9990 & 1.14 & 3.80 \\
pCA & $10-150$ & 0.0211 & 0.0061 & 0.9987 & 1.82 & 6.07 \\
TCNE & $5-60$ & 0.0062 & 0.0183 & 0.9989 & 4.32 & 14.39 \\
Bromanil & $25-100$ & 0.0074 & 0.0087 & 0.9993 & 5.11 & 17.02 \\
Chloranil & $40-200$ & 0.0171 & 0.0021 & 0.9984 & 12.24 & 40.76 \\
\hline
\end{tabular}

Table 3. Precision of the proposed methods for determination of ROS-Ca based on its CT reaction with different acceptors.

\begin{tabular}{|c|c|c|c|c|c|}
\hline \multirow[b]{2}{*}{$\begin{array}{l}\text { Acceptor-based } \\
\text { method }\end{array}$} & \multirow[b]{2}{*}{$\begin{array}{c}\text { ROS-Ca } \\
\left(\mu \mathrm{g} \mathrm{mL} \mathbf{~}^{-1}\right)\end{array}$} & \multicolumn{2}{|c|}{ Within-assay, $n=6$} & \multicolumn{2}{|c|}{ Between-assays, $n=6$} \\
\hline & & 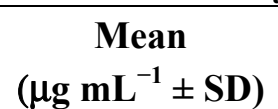 & RSD & 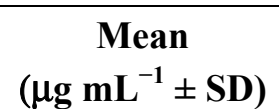 & RSD \\
\hline $\mathrm{pCA}$ & 50 & $48.78 \pm 0.41$ & 0.84 & $50.05 \pm 0.84$ & 1.68 \\
\hline DDQ & 20 & $20.51 \pm 0.25$ & 1.22 & $19.56 \pm 0.36$ & 1.84 \\
\hline TCNE & 40 & $40.57 \pm 0.75$ & 1.85 & $38.96 \pm 0.75$ & 1.93 \\
\hline TNB & 20 & $20.73 \pm 0.23$ & 1.11 & $19.55 \pm 0.35$ & 1.79 \\
\hline TCNQ & 40 & $38.78 \pm 0.16$ & 0.41 & $38.04 \pm 0.29$ & 0.76 \\
\hline Bromanil & 80 & $81.57 \pm 0.84$ & 1.03 & $78.24 \pm 0.81$ & 1.04 \\
\hline Chloranil & 200 & $198.78 \pm 2.69$ & 1.35 & $201.05 \pm 2.08$ & 1.03 \\
\hline
\end{tabular}




\subsubsection{Ruggedness and Robustness}

The ruggedness of the proposed methods was assessed by applying the procedures using two different instruments (shown in the Experimental section) in two different laboratories at different elapsed time. Results obtained from lab-to-lab and day-to-day were found to be reproducible as RSD did not exceed $2 \%$. Robustness of the procedures was assessed by evaluating the influence of small variation of experimental variables: concentrations of acceptor reagent, and reaction time, on the analytical performance of the method. In these experiments, one experimental parameter was changed while the other parameters were kept unchanged, and the recovery percentage was calculated each time. The small variations in any of the variables did not significantly affect the results; recovery percentages were $97.25-103.51 \% \pm 0.74-1.92 \%$. This provided an indication for the reliability of the proposed methods during routine work.

\subsection{Application of the Method to the Analysis of Tablets}

The obtained satisfactory validation results made the proposed procedures suitable for the routine quality control analysis of ROS-Ca. The proposed and the reported method [14] were applied to the determination of ROS-Ca in its tablets. The results obtained by the proposed methods were statistically compared with those obtained by the reported method. The obtained values of the labeled amount were 99.54-100.46 $\pm 1.58-1.82 \%$ (Table 4 ). In the $t$ - and F-tests, no significant differences were found between the calculated and theoretical values of both the proposed and the reported methods at $95 \%$ confidence level. This indicated similar precision and accuracy in the analysis of ROS-Ca in its capsules. It is evident from these results that all the proposed methods are applicable to the analysis of ROS-Ca in its pharmaceutical tablets with comparable analytical performance. However, the critical recommendations of some of these methods might be based on the experimental conditions (e.g., reaction time), and the ultimate sensitivity that determines the amount of specimen required for analysis. For example, the methods involving DDQ, pCA, bromanil, and chloranil are recommended whenever rapid analysis is required; this because they have very short reaction time. The method involving TCNQ is recommended, as high sensitivity is required on the expense of the analysis time.

Table 4. Determination of ROS-Ca in its tablets by the proposed methods.

\begin{tabular}{|c|c|c|c|}
\hline Method & Label claim $(\% \pm \mathrm{SD})^{\mathrm{a}}$ & t-values ${ }^{b}$ & F-values ${ }^{b}$ \\
\hline $\mathrm{pCA}$ & $99.58 \pm 1.77$ & 1.55 & 2.41 \\
\hline DDQ & $100.21 \pm 1.43$ & 1.26 & 1.57 \\
\hline TCNE & $99.54 \pm 1.58$ & 1.86 & 1.92 \\
\hline TNB & $99.82 \pm 0.78$ & 0.83 & 0.47 \\
\hline TCNQ & $100.09 \pm 1.96$ & 0.61 & 2.20 \\
\hline Bromanil & $100.29 \pm 1.32$ & 1.72 & 1.34 \\
\hline Chloranil & $100.46 \pm 1.82$ & 2.10 & 2.55 \\
\hline Reported $^{\mathrm{c}}$ & $99.95 \pm 1.14$ & & \\
\hline
\end{tabular}

${ }^{a}$ Values are mean of five determinations. ${ }^{\mathrm{b}}$ The tabulated values of $\mathrm{t}$ and $\mathrm{F}$ at $95 \%$ confidence limit are 2.78 and 6.39, respectively. ${ }^{\mathrm{c}}[14]$. 


\section{Experimental Section}

\subsection{Apparatus}

Double beam ultraviolet-visible spectrophotometer with matched 1-cm quartz cells (UV-1601 PC; Shimadzu, Kyoto, Japan) was used for all the spectrophotometric measurements.

\subsection{Chemicals and Reagents}

ROS-Ca was obtained from Pfizer Inc. (New York, NY, USA). Ezetimibe was obtained from AK Scientific Inc. (30023 Ahern Avenue Union City, CA, USA). Fenofibrate was obtained from Sigma Chemical Co. (St. Louis, MO, USA). The purities of the investigated compounds were $>99 \%$, and the solutions were stable for at least one week when kept refrigerated). 7,7,8,8-tetracyanoquinodimethane (TCNQ; Aldrich Chem. Co, Milwaukee, WI, USA) was $1 \mathrm{mg} \mathrm{mL}^{-1}$ in acetonitrile, and the solution was stable for at least 1 week at $4{ }^{\circ} \mathrm{C}$. 1,3,5-Trinitrobenzene (TNB; Prodotti Chimiol Pet Analist Scientifico, Milano, Italy) was $4 \mathrm{mg} \mathrm{mL}^{-1}$ in acetonitrile, and the solution was prepared fresh daily. 2,3-Dichloro-5,6-dicyano-1,4-benzoquinone (DDQ; Merck Schuchardt, Munich, Germany) was $2 \mathrm{mg}$ $\mathrm{mL}^{-1}$ in methanol and it was prepared fresh daily. 2,5-Dichloro-3,6-dihydroxy-1,4-benzoquinone (chloranilic acid, pCA) from BDH Chemicals (Poole, UK) was $4 \mathrm{mg} \mathrm{mL}^{-1}$ in acetonitrile, and the solution was prepared fresh daily. Tetracyanoethylene (TCNE; Nacalai Tesque, Kyoto, Japan) was $1 \mathrm{mg} \mathrm{mL}{ }^{-1}$ in acetonitrile, and it was prepared fresh daily. 2,3,5,6-Tetrabromo-1,4-benzoquinone (bromanil; Hopkin \& Williams Ltd, Heneage Street, Birmingham, UK), and 2,3,5,6-tetrachloro-1,4benzoquinone (chloranil; Sigma Chemical Co. 3050 Spruce St St Louis, MO 63103, USA) were $5 \mathrm{mg} \mathrm{mL}{ }^{-1}$ in acetonitrile, and the solutions were prepared fresh daily. 2,4,7-Trinitro-9-fluorenone (TNF; Fluka, Buchs, Switzerland) was $5 \mathrm{mg} \mathrm{mL}^{-1}$ in acetonitrile and the solution was prepared fresh daily. Crestor tablets (Astra Zeneca Pharma, Bengaluru, India) labeled to contain $5 \mathrm{mg}$ ROS-Ca were obtained from the market. All solvents and other chemicals used throughout this study were of analytical grade.

\subsection{Preparation of Standard and Tablets Sample Solutions}

\subsubsection{Preparation of Stock Standard ROS-Ca Solution}

Into a 50-mL calibrated flask, ROS-Ca (100 mg) was accurately weighed and dissolved in methanol ( $2 \mathrm{~mL}$ ), completed to volume with methanol for DDQ and with acetonitrile for the other acceptors. This stock solution $\left(2 \mathrm{mg} \mathrm{mL} \mathrm{m}^{-1}\right)$ was diluted with the respective solvents to obtain suitable concentrations that lie in the linear range of each particular assay method.

\subsubsection{Preparation of Tablets Sample Solution}

Twenty tablets were weighed and finely powdered. A quantity of the powder equivalent to $50 \mathrm{mg}$ of ROS-Ca was transferred into a 25-mL calibrated flask, dissolved in methanol $(2 \mathrm{~mL})$, swirled and sonicated for $5 \mathrm{~min}$, completed to volume with the corresponding solvent (as in stock solution), shaken well for $15 \mathrm{~min}$, and filtered. The first portion of the filtrate was rejected, and a measured volume of 
the filtrate was diluted quantitatively with a suitable solvent to yield suitable concentrations as the linear range of each particular assay method.

\subsection{General Analytical Procedure}

One milliliter of the standard or sample solution of ROS-Ca $\left(20-2,000 \mu \mathrm{g} \mathrm{mL}^{-1}\right)$ was transferred into 10-mL calibrated flasks. One milliliter of the acceptor solution was added, and the reaction was allowed to proceed at room temperature $\left(25 \pm 2{ }^{\circ} \mathrm{C}\right.$ ) for $5 \mathrm{~min}$ (in case of pCA, DDQ, bromanil and chloranil), $15 \mathrm{~min}$ (in case of TCNQ and TCNE), $30 \mathrm{~min}$ (in case of TNB), and for $60 \mathrm{~min}$ (in case of TNF). The solutions were diluted to volume with methanol for DDQ and with acetonitrile for the other acceptors. The absorbances of the resulting solutions were measured at the wavelengths of maximum absorption (840, 435, 460, 518, 412, 498, 460, and $412 \mathrm{~nm}$ for TCNQ, TNB, DDQ, pCA, TCNE, bromanil, chloranil, and TNF, respectively) against reagent blanks treated similarly.

\subsection{Determination of Molar Ratio}

The Job's method of continuous variation [34] was employed. Master equimolar solutions of ROS-Ca and reagents were prepared. The concentrations of these solutions were $4.9 \times 10^{-3} \mathrm{M}$ (in acetonitrile for TCNQ), $1.9 \times 10^{-2} \mathrm{M}$ (in acetonitrile for TNB), $8.8 \times 10^{-3} \mathrm{M}$ (in methanol for DDQ), $1.9 \times 10^{-2} \mathrm{M}$ (in acetonitrile for $\mathrm{pCA}$ ), $1.6 \times 10^{-2} \mathrm{M}$ (in acetonitrile for TCNE), $1.2 \times 10^{-2} \mathrm{M}$ (in acetonitrile for bromanil), and $2 \times 10^{-2} \mathrm{M}$ (in acetonitrile for chloranil). Series of 10-ml portions of the master solutions of ROS-Ca with the respective reagent were made up comprising different complementary proportions $(0: 10,1: 9, \ldots \ldots . ., 9: 1,10: 0$, inclusive $)$ in 10-mL calibrated flasks. The reactions were allowed to proceed for the optimum reaction time (Table 1) and then the absorbances of the resulting solutions were measured at the corresponding wavelengths of maximum absorbances $\left(\lambda_{\max }\right)$.

\subsection{Molecular Modeling for the CT Complex of ROS-Ca with DDQ}

The molecular modeling for the CT complex of ROS-Ca with DDQ was performed by using CS Chem3D Ultra, version 9 (Cambridge Soft Corporation, Cambridge, MA, USA) implemented with molecular orbital computations software (MOPAC), and molecular dynamics computations software.

\section{Conclusions}

The CT reaction of ROS-Ca as electron donor and some $\pi$-electron acceptors has been investigated. The obtained complexes were studied by ultraviolet-visible spectrophotometry. The obtained colored CT complexes were utilized in the development of seven simple, rapid and accurate spectrophotometric methods for the analysis of ROS-Ca in pure form as well as in capsules. The methods described herein have many advantages: they do not need expensive sophisticated apparatus, they are simple and rapid, and they have high sensitivity. The proposed methods used inexpensive reagents with excellent shelf life, and are available in any analytical laboratories. Therefore, the methods are practical and valuable for routine application in quality control laboratories for analysis of ROS-Ca. 


\section{Acknowledgments}

The authors would like to extend their appreciation to the Deanship of Scientific Research at King Saud University for its funding of this research through the research group project No. RGP-VPP-225.

\section{Conflict of Interest}

The authors declare no conflict of interest.

\section{References}

1. Cheng-Lai, A. Rosuvastatin: A new HMG-CoA reductase inhibitor for the treatment of hypercholesterolemia. Heart Dis. 2003, 5, 72-78.

2. Cheng, J.W. Rosuvastatin in the management of hyperlipidemia. Clin. Ther. 2004, 26, 1368-1387.

3. Wani, T.A.; Samad, A.; Tandon, M.; Saini, G.S.; Sharma, P.L.; Pillai, K.K. The effects of rosuvastatin on the serum cortisol, serum lipid, and serum mevalonic acid levels in the healthy Indian male population. AAPS Pharm. Sci. Tech. 2010, 11, 425-432.

4. Chaudhari, B.G.; Patel, N.M.; Shah, P.B. Determination of simvastatin, pravastatin sodium and rosuvastatin calcium in tablet dosage forms by HPTLC. Indian J. Pharm. Sci. 2007, 69, 130-132.

5. Kaila, H.O.; Ambasana, M.A.; Thakkar, R.S.; Saravaia, H.T.; Shah, A.K. A new improved RP-HPLC method for assay of rosuvastatin calcium in tablets. Indian J. Pharm. Sci. 2010, 72, 592-598.

6. Pandya, C.B.; Channabasavaraj, K.P.; Chudasama, J.D.; Mani, T.T. Development and validation of RP-HPLC method for determination of rosuvastatin calcium in bulk and pharmaceutical dosage form. Inter. J. Pharm. Sci. Rev. Res. 2010, 5, 82-86.

7. Suslu, I.; Çelebier, M.; Altınoz, S. Determination of rosuvastatin in pharmaceutical formulations by capillary zone electrophoresis. Chromatographia 2007, 66, 65-72.

8. Darwish, I.A.; Sultan, M.A.; Al-Arfaj, H.A. Novel selective kinetic spectrophotometric method for determination of norfloxacin in its pharmaceutical formulations. Talanta. 2009, 78, $1383-1388$.

9. Darwish, I.A.; Abdine, H.H.; Amer, S.M.; Al-Rayes, L.I. Spectrophotometric study for the reaction between fluvoxamine and 1,2-naphthoquinone-4-sulphonate: Kinetic, mechanism and use for determination of fluvoxamine in its dosage forms. Spectrochim. Acta. A 2009, 72, 897-902.

10. Darwish, I.A.; Hussein, S.A.; Mahmoud, A.M.; Hassan, AI. Spectrophotometric determination of $\mathrm{H}(2)-r e c e p t o r$ antagonists via their oxidation with cerium(IV). Spectrochim. Acta A 2008, 69, 33-40.

11. Ahmed, S.; Rasul, A.; Masood, Z. Spectrophotometry in Pharmaceutical Analysis; LAP Lambert Academic Publishing: Saarbrucken, Germany, 2011.

12. Gorog, S. Ultraviolet-visible Spectrophotometry in Pharmaceutical Analysis; CRC Press: Boca Raton, FL, USA, 1995.

13. Darwish, I.A.; Refaat, I.H.; Askal, H.F.; Marzouq, M.A. Generic nonextractive spectrophotometric method for determination of 4-quinolone antibiotics by formation of ion-pair complexes with beta-naphthol. J. AOAC Int. 2006, 89, 334-340. 
14. Uyar, B.; Celebier, M.; Altinoz, S. Spectrophotometric determination of rosuvastatin calcium in tablets. Pharmazie 2007, 62, 411-413.

15. Sevda, R.R.; Ravetkar, A.S.; Shirote, P.J. UV Spectrophotometric estimation of rosuvastatin calcium and fenofibrate in bulk drug and dosage form using simultaneous equation method. Int. J. Chem. Tech. Res. 2011, 3, 629-635.

16. Gupta, A.; Mishra, P.; Shah, K. Simple UV spectrophotometric determination of rosuvastatin calcium in pure form and in pharmaceutical formulations. E-J. Chem. 2009, 6, 89-92.

17. Marothu, V.K.; Dannana, G.S. Extractive spectrophotometric methods for the determination of rosuvastatin calcium in pure form and in pharmaceutical formulations by using Safranin $\mathrm{O}$ and Methylene blue. E-J. Chem. 2007, 4, 46-49.

18. Darwish, I.A. Development and validation of spectrophotometric methods for determination of fluoxetine, sertraline, and paroxetine in pharmaceutical dosage forms. J. AOAC Int. 2005, 88, 38-45.

19. Darwish, I.A.; Wani, T.A.; Khalil, N.Y.; Al-Shaikh, A.A.; Al-Morshadi, N. Development of a novel 96-microwell assay with high throughput for determination of olmesartan medoxomil in its tablets. Chem. Cent. J. 2012, 6, 1.

20. Wani, T.A.; Khalil, N.Y.; Abdel-Rahman, H.M.; Darwish, I.A. Novel microwell-based spectrophotometric assay for determination of atorvastatin calcium in its pharmaceutical formulations. Chem. Cent. J. 2011, 5, 57.

21. Darwish, I.A. Kinetic spectrophotometric assays for determination of trimetazidine dihydrochloride. Anal. Chim. Acta 2005, 551, 222-231.

22. Darwish, I.A. Analytical study for the charge transfer complexes of losartan potassium. Anal. Chim. Acta 2005, 549, 212-220.

23. Darwish, I.A. Refaat, I.H. Spectrophotometric analysis of selective serotonin reuptake inhibitors based on formation of charge-transfer complexes with tetracyanoquinodimethane and chloranilic acid. J. AOAC Int. 2006, 89, 326-333.

24. Darwish, I.; Abdel-Wadood, H.; Abdel-Latif, N. Validated spectrophotometric and fluorimetric methods for analysis of clozapine in tablets and urine. Ann. Chim. 2005, 95, 345-356.

25. Huang, W.; Liu, X.J.; Zhao, F.L. Spectrophotometric determination of azithromycin by charge transfer reaction. Chin. J. Mod. Appl. Pharm. 2006, 26, 913-916.

26. Taha, A.; Rucker, G. Utility of pi-acceptors in alkaloid assay. Arch. Pharm. (Weinheim.) 1977, 310, 485-494.

27. Melby, L.R. The Chemistry of the Cyano Group; John Wiley \& Sons: New York, NY, USA, 1970; pp. 656-659.

28. Yamagishi, A. Solvation effects on the electron-transfer reaction of TCNQ anion radical and 2,3-dichloro-5, 6-dicyano-p-benzoquinone. Bull. Soc. Jpn. 1975, 48, 2440.

29. Foster, R. Organic Charge-Transfer Complexes; Academic Press: New York, NY, USA, 1969; p. 470.

30. Vogel's Textbook of Practical Organic Chemistry, 5th ed.; Longman group UK Ltd.: London, UK, 1989; pp. 1442-1444.

31. The United States Pharmacopeia 24, The National Formulary 19; United States Pharmacopeial Convention, Inc.: Rockville, ML, USA, 2008. 
32. Agouridis, A.P.; Tsimihodimos, V.; Filippatos, T.D.; Tselepis, A.D.; Elisaf, M.S. High doses of rosuvastatin are superior to low doses of rosuvastatin plus fenofibrate or n-3 fatty acids in mixed dyslipidemia. Lipids 2011, 46, 521-528.

33. Kawashiri, M.A.; Nohara, A.; Noguchi, T.; Tada, H.; Nakanishi, C.; Mori, M.; Konno, T.; Hayashi, K.; Fujino, N.; Inazu, A.; et al. Efficacy and safety of coadministration of rosuvastatin, ezetimibe, and colestimide in heterozygous familial hypercholesterolemia. Am. J. Cardiol. 2012, 109, 364-369.

34. Job, P. Advanced Physicochemical Experiments, 2nd ed.; Oliner and Boyd: Edinburgh, UK, 1964; p. 54.

Sample Availability: Not available.

(C) 2013 by the authors; licensee MDPI, Basel, Switzerland. This article is an open access article distributed under the terms and conditions of the Creative Commons Attribution license (http://creativecommons.org/licenses/by/3.0/). 